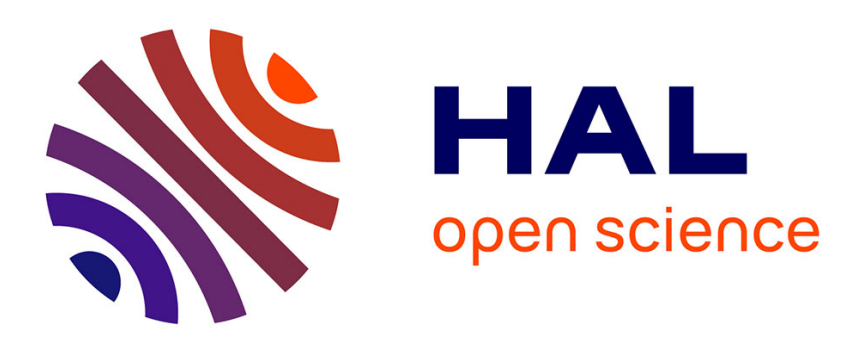

\title{
Estimation of arterial wall motion using ultrafast imaging and transverse oscillations: in vivo study
}

Sébastien Salles, Anne Long, Lorena Petrusca, Vincent Perrot, Didier Vray, Hervé Liebgott

\section{- To cite this version:}

Sébastien Salles, Anne Long, Lorena Petrusca, Vincent Perrot, Didier Vray, et al.. Estimation of arterial wall motion using ultrafast imaging and transverse oscillations: in vivo study. IEEE International Ultrasonics Symposium (IUS 2016), Sep 2016, Tours, France. 10.1109/ULTSYM.2016.7728749 . hal01438446

\author{
HAL Id: hal-01438446 \\ https://hal.science/hal-01438446
}

Submitted on 11 Mar 2019

HAL is a multi-disciplinary open access archive for the deposit and dissemination of scientific research documents, whether they are published or not. The documents may come from teaching and research institutions in France or abroad, or from public or private research centers.
L'archive ouverte pluridisciplinaire HAL, est destinée au dépôt et à la diffusion de documents scientifiques de niveau recherche, publiés ou non, émanant des établissements d'enseignement et de recherche français ou étrangers, des laboratoires publics ou privés. 


\section{Estimation of arterial wall motion using ultrafast imaging and transverse oscillations: in vivo study}

\author{
Sebastien Salles \\ Department of Circulation and \\ Medical Imaging, Norwegian \\ University of Science and \\ Technology, Norway
}

\author{
Anne Long \\ Univ Lyon, INSA-Lyon, \\ Université Lyon 1, CNRS, Inserm, \\ CREATIS UMR 5220, U1206, F- \\ 69621. \\ Department of Internal Medicine \\ and Vascular Medicine, Hospices \\ Civils de Lyon, University \\ Hospital Edouard Herriot, Place \\ d'Arsonval, 69437 Lyon 1 \\ University, Lyon, France LYON, \\ France
}

Lorena Petrusca

Univ Lyon, INSA-Lyon,

Université Lyon 1, UJM-Saint

Etienne, CNRS, Inserm,

CREATIS UMR 5220, U1206, F-

42023, Saint-Etienne, France

\author{
Vincent Perrot, Didier Vray, \\ Herve Liebgott \\ Univ Lyon, INSA-Lyon, \\ Université Lyon 1, CNRS, Inserm, \\ CREATIS UMR 5220, U1206, F- \\ 69621, LYON, France
}

\begin{abstract}
Early detection of cardiovascular diseases can be done by assessing the dynamic properties of arteries. In this study, two phenomena related to the carotid wall motion are presented. The 2D motion estimation method employs (i) ultrafast imaging which became a world-wide use modality, (ii) transverse oscillations technics which allow improving the motion estimation in transverse direction i.e. perpendicular to the beam, and (iii) a 2D phase shift estimator. First, using only the radial acceleration wall, the Wall Spatial Periodic Ripples (WSPR) is studied under a cold pressor test. The results show that the WSPR mean maximum amplitude decreased of $22.9 \%$ at the systolic wave and $33.2 \%$ at the dicrotic notch. So, the WSPR is a possible artery wall rigidity marker. Then, using a very high frame rate imaging, the longitudinal carotid wall motion of one healthy subject is studied. This second experimentation permits to estimate the propagation of a longitudinal motion on an in vivo carotid. Its mean velocity was evaluated at $16.1 \mathrm{~m} . \mathrm{s}^{-1}$, which given a ratio of 3 between with the estimated PWV.

Keywords-Ultrafast imaging, Transverse oscillations, Carotid, Wall spatial periodic ripples, Waves propagation.
\end{abstract}

\section{INTRODUCTION}

Ultrafast ultrasound is a promising imaging modality with several potential clinical applications, such as arterial rigidity assessment [1], heart motion [2] and blood flow imaging [3]. Ultrafast ultrasound allows one to examine phenomena never analyzed before. Over the past decade, researchers have shown interest to study the carotid wall motion, especially the longitudinal motion of the intima-media complex (IMC), to improve vascular pathology diagnosis [4], [5]. Under this motivation we are interested in studying the instantaneous 2D vector motion and trajectories of the carotid artery wall, in ultrafast imaging.

Ultrafast imaging has already been applied on the carotid artery, in combination with acoustic radiation force for shear wave imaging [6], [7], or to image the natural pulse wave locally. The pulse wave velocity (PWV) of the carotid artery has been quantified and has proved to be an excellent indicator of cardiovascular diseases [8], [9]. However, to the best of our knowledge, only the waves velocities are extracted and used to assess the elastic parameters, the spatial wall behavior, i.e the motion variation along the wall over a given time, has never been studied. The first objective of this paper was to study the wall spatial periodic ripples (WSPR) in high frame rate imaging (2500 frames per second, FPS), and to evaluate its amplitude as stiffness wall marker using a cold pressor test.

In the literature the pulse wave propagation studies focused only on the propagation of the axial displacement of the artery wall, and most of them were not specific to the IMC. Consequently, the longitudinal motion of the IMC has never been studied in ultrafast imaging. Indeed, probably some phenomena which are unobservable with conventional imaging techniques are likely to occurred in the longitudinal direction of this complex and might be imaged and quantified with specific imaging techniques. Especially, a second wave, corresponding to the propagation of a longitudinal motion, was identified and evaluated in the 1960s [10], [11]. Called "Type II wave", "Lamb mode" or bulk wave (BW), its velocity (BWV) was estimated three times greater than the velocity of the pulse wave in the carotid artery of the dog in situ [12]. The second objective of this paper was to study the longitudinal wall motion in very high frame rate imaging (15 000 FPS) in order to visualize and to evaluate the velocity of this bulk wave in the carotid in vivo.

Because the longitudinal motion estimation of the IMC is challenging, we have previously developed vector motion estimation methods based on transverse oscillations (TO) technique. This method, initially developed for vector flow imaging [13], [14] in medical ultrasound, has been adapted for tissue vector motion estimation by our group [15], [16] and others [17], [18]. The idea behind TO is to produce a pressure field featuring oscillations in the axial direction, i.e. along the ultrasound beam axis, and also in the perpendicular direction. The objective is to generate ultrasound images suitable for transverse motion estimation, which is typically not the case with conventional images. TO schemes are usually combined with phase-based motion estimation techniques [15], [16]. The 
validation and the feasibility of this method have already been done in conventional imaging [19], [20] and in 3D imaging [21]. This method was also validated in ultrafast imaging [22] where the PWV, and the Young's modulus of the artery phantom were estimate accurately with a mean errors of $2.2 \%$.

In the following, section II presents the experimental setups and briefly recalls the principles of our techniques. Then, in section III, two experimental results are presented. Firstly, acquisitions at high frame rate are performed on 8 healthy subjects before and after a cold pressor test in order to evaluate the WSPR maximum amplitude as stiffness wall marker. And then, a one case study is presented, where very high frame rate imaging is performed on a healthy subject in order to study the longitudinal motion of the IMC.

\section{MATERIAL AND METHOD}

\section{A. Data Acquisition}

For data acquisition, a Verasonics ultrasound system (Verasonics Inc., Redmond, WA) with the L7-4 probe were used in this study. One common way to achieve ultrafast imaging is to produce plane waves by exciting all the probe elements simultaneously. Only a single plane wave insonification at $90^{\circ}$ was transmitted. The raw RF data were beamformed using the Stolt's method for plane wave imaging [23]. The linear L7-4 array used and the acquisition parameters are described in Tab. I.

Tab. I. Acquisition parameters

\begin{tabular}{ll}
\hline \hline Parameter & Value \\
\hline Linear array & \\
Pitch & $296 \mu \mathrm{m}$ \\
Center frequency & $5.9 \mathrm{MHz}$ \\
\hline Imaging parameters & $5 \mathrm{MHz}$ \\
Transmit center frequency & $1-$ cycle sinusoidal pulse \\
Transmit pulse & $25 \mathrm{MHz}$ \\
Sampling frequency & $30 \mathrm{~mm}$ \\
Max imaging depth & $2.5 \mathrm{kHz}$ \\
PRF & $15 \mathrm{kHz}$ \\
Steering angle & $0^{\circ}$ \\
Transmit aperture & 128 elements \\
Frame Rate & First experiment: $2.5 \mathrm{kHz}$ \\
& Second experiment: $15 \mathrm{kHz}$ \\
\hline \hline
\end{tabular}

First experiment: The longitudinal (long-axis) view of the right common carotid arteries of $8(n=8)$ healthy subjects was scanned, at 2500 FPS, during 3 s to include 4 cardiac cycles before and just after a cold pressor test. This test consists in immersing the right hand in cold water $\left(4-10^{\circ} \mathrm{C}\right)$ during 3 min and has for effect to increase heart reactivity (blood pressure, heart rate) and the PWV [24], [25]. Thanks to that we will be able to see if the WSPR can be seen as stiffness wall marker by performing a relative difference between its maximum amplitude obtained before and after the cold pressor test.

Second experiment: The longitudinal (long-axis) view of the right common carotid arteries of one healthy subject was scanned at $15000 \mathrm{FPS}$, during 1s, and 3 times in order to have 3 cardiac cycles. This frame rate was chosen in order to visualize the longitudinal motion with a good temporal resolution.

\section{B. Data Processing}

Motion estimation using Transverse Oscillations: The carotid wall displacement was estimated combining a Phase Based Motion Estimation (PBME) and TO. The TO was introduced by filtering the beamformed RF image

[22]. In this study the TO wavelength was $2 \mathrm{~mm}$. It then becomes possible to estimate the $2 \mathrm{D}$ vector motion using the phase-based technique described in [15]. The radial and lateral windows size was equal to $1 \mathrm{~mm}$ and $1.4 \mathrm{~mm}$, respectively

First experiment: The local wall displacements between two consecutive images were estimated, at each location within and along the wall, using the before-mentioned method. Then, the distal wall radial acceleration was extracted, and the amplitude of the WSPR was estimated by removing the global wall acceleration (Fig. 1). Its maximum amplitude was compared to the maximum amplitude of the carotid diameter variation, the longitudinal wall motion, and the regional PWV, by averaging over 4 cardiac cycles. Because of the difficulty to visualize the IMC in the proximal wall, the longitudinal wall motion, and the regional PWV was estimated only in the distal wall. Finally, the relative differences between the values estimated before and after the cold-pressor were computed and the mean of these differences was calculated (Tab. II).

Second experiment: The distal wall local velocity was estimated between each consecutive 50 images $\left(I_{1} \rightarrow I_{51}\right.$, $\mathrm{I}_{2} \rightarrow \mathrm{I}_{52}$, etc.) at each location within and along the wall. The wave velocity was estimating at the dicrotic notch time, thanks to the foot (i.e., fiduciary point) of the wall velocity waveform. It was defined as the inflection point where the temporal derivative of the velocity (i.e., wall acceleration) attains its maximum. In this study, as the longitudinal acceleration being noisier, the wave velocity was estimated by taking the centroid of the acceleration front (Fig. 2).

\section{RESULTS}

\section{First experiment:}

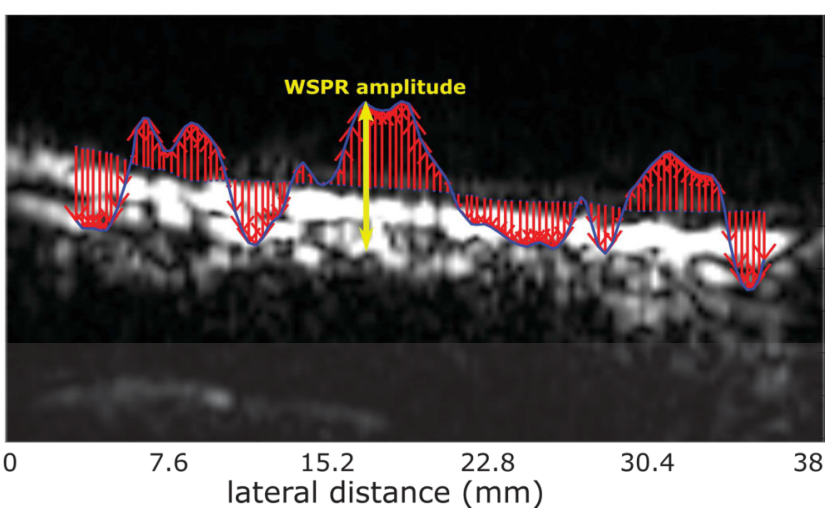

Fig. 1: Investigated distal wall of a carotid artery, the red vectors represent the axial acceleration of the wall. The WSPR is represented with the blue line.

We observed and measured WSPR all along the carotid artery (Fig.1). The estimated WSPR amplitude before the cold pressor test is represented Fig. 2a. WSPR amplitude was 
consistent and reproducible according 4 cardiac cycles. For each cardiac cycle two maximum peaks were visible. By looking at the related radial wall velocity, we noticed that the first peak corresponds to the systolic wave and the second one corresponds to the dicrotic notch wave, which led us to think that the WSPR is due to the propagating waves.

The WSPR after the cold pressor test is depicted Fig. 2b. Its amplitude clearly decreased after the cold pressor test, specifically only the two maximum peaks decreased, the rest of the signal seemed to stay the same.
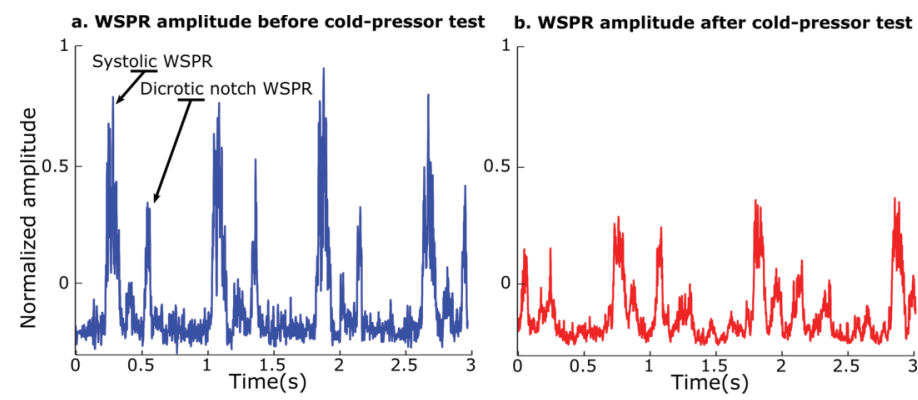

Fig. 2: WSPR amplitude of the wall, before and after the cold-pressor over 4 cardiac cycle, respectively. The amplitude clearly decreases after the cold-pressor test.

The mean value, of the relative differences, between the stiffness markers described in section II.B obtained before and after the cold pressor test calculated of all our population are depicted in Tab. II.

First, the results showed that the cold-pressor test affects significantly the wall stiffness since the maximum amplitude of the carotid diameter and the longitudinal motion of the wall motions decreased, and PWV increased. Moreover, we have noticed that the dicrotic notch phase seemed more affected than the systolic one.

Tab. II: Mean of the relative differences between before and after the cold-pressor test

\begin{tabular}{|l|c|}
\hline Diameter maximum amplitude & $-4.9 \%$ \\
\hline Longitudinal motion maximum amplitude & $-10.4 \%$ \\
\hline Systolic PWV & $11.9 \%$ \\
\hline Dicrotic notch PWV & $22.9 \%$ \\
\hline Systolic WSPR maximum amplitude & $-12.9 \%$ \\
\hline Dicrotic notch WSPR maximum amplitude & $-35.7 \%$ \\
\hline
\end{tabular}

Then, according to those results the WSPR maximum amplitude decreases in the same order of magnitude as the PWV, with a slightly higher relative difference for the dicrotic notch WSPR maximum amplitude.

Those results conduct us to think that the WSPR amplitude could be a new stiffness marker.

\section{Second experiment:}

Fig. 3 illustrates the spatiotemporal variation of the wall radial and longitudinal acceleration of the two first acquisitions of the same healthy subject. Only the region around the dicrotic notch was studied and shown. The radial and longitudinal acceleration pattern was found consistent and reproducible between each acquisition. The wave propagation velocities were estimated at the first positive transition by estimating the centroid of the acceleration waveform. The green lines correspond to the two foots of the accelerations, which was estimated by looking for the maximum value of the acceleration divided by 2 . Then, the centroid between the two previously estimated values was extracted.

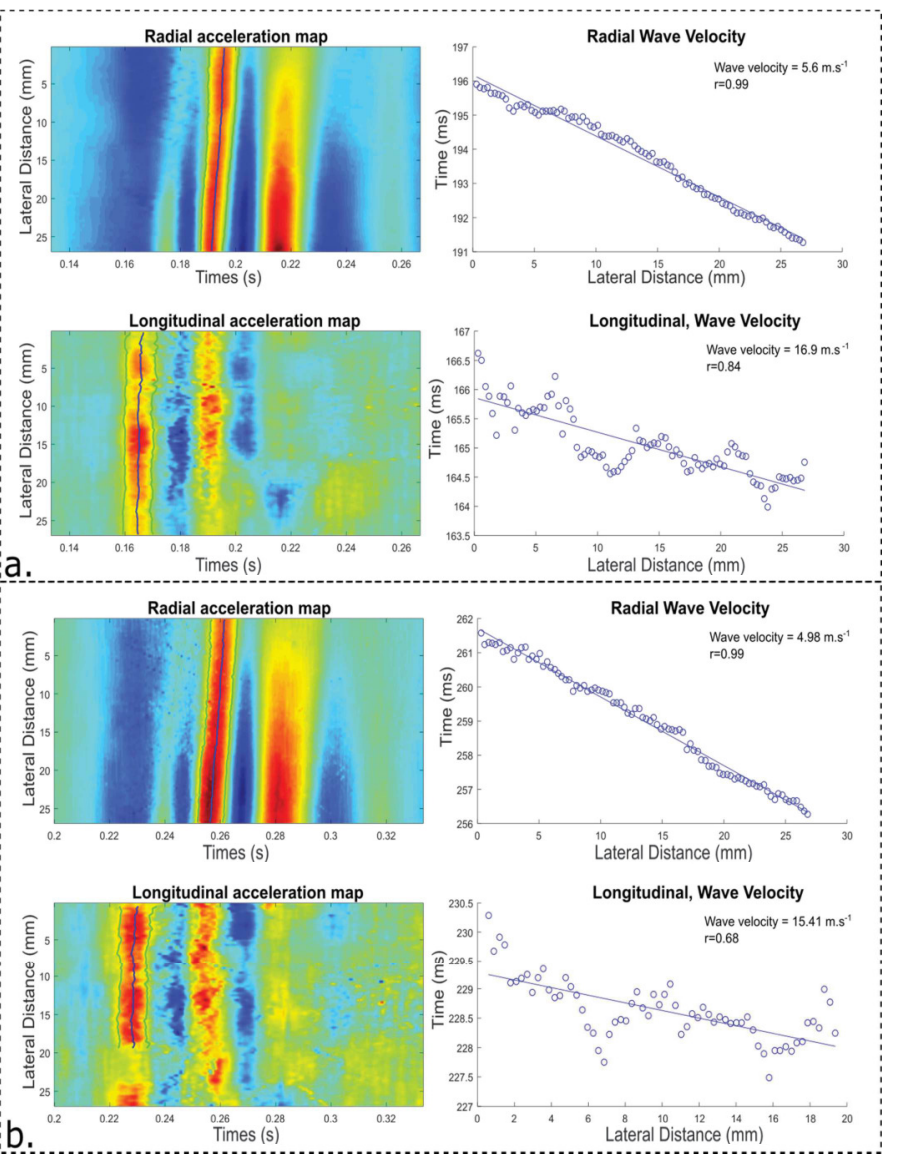

Fig. 3: Spatiotemporal variation of the radial and longitudinal wall acceleration in the carotid artery at the dicrotic notch. (a) and (b) represent two different acquisitions of the same subject. The green lines correspond to the two foots of the accelerations, while the blue line shown the centroid of the front. On the right the time versus distance of the pulse wave propagation and the linear regression are shown.

The time of the centroid was plotted against the distance travelled by the pulse wave. A linear regression fit was applied on the time-distance plot. The wave velocities were calculated as the reciprocal of the linear regression slope, and reported on Tab. III. As expected, the mean estimated PWV was $5.3 \mathrm{~m} . \mathrm{s}^{-1}$, which is the typical value found in the literature for the dicrotic notch. The Fig. 3 clearly exhibits a propagation of a longitudinal motion which was assumed to be the BW. Moreover, a time shift was visible between the first positive transition of the radial and longitudinal acceleration. Concerning the Fig. 3b, since the estimated longitudinal acceleration wasn't continued along the wall, the BWV was estimated on a piece of the map. The mean estimated BWV was $16.1 \mathrm{~m} . \mathrm{s}^{-1}$ with a mean correlation coefficient (r) of 0.78 . A ratio of 3 was estimated between the PWV and BWV which corresponds to the ratio given in [12]. 


\begin{tabular}{|l|c|c|c|}
\hline \multicolumn{2}{|l|}{ Tab. III: Waves Velocity } & \\
\hline Acquisition & PWV $\left({\left.\mathrm{m} . \mathrm{s}^{-1}\right),[\mathrm{r}]}\right.$ & $\mathrm{BWV}\left({\left.\mathrm{m} . \mathrm{s}^{-1}\right),[\mathrm{r}]}\right.$ & Ratio \\
\hline 1 & $5.6[0.99]$ & $16.9[0.84]$ & 3.01 \\
\hline 2 & $5[0.99]$ & $15.4[0.68]$ & 3.08 \\
\hline 3 & $5.4[0.99]$ & $16[0.82]$ & 2.96 \\
\hline
\end{tabular}

Several questions came up concerning this BW. What is the generation mechanism of this wave? Where does the time shift come from? Can we use this wave to better understand the longitudinal motion of the carotid? And finally, can we link the BWV to the wall arterial stiffness? We will try to answers these questions by studying a larger population of healthy subjects.

\section{CONCLUSION}

In this work, the 2D motion of healthy carotid wall was studied with high frame rate ultrasound imaging through two experiments at different FPS. First, using a high frame rate imaging at 2500 FPS, the carotid WSPR in the wake of the PW was highlighted and studied under a cold-pressor test. We have shown that the WSPR could be seen as wall rigidity marker since its maximum amplitude is related to the arterial stiffness. Indeed, the mean relative difference WSPR maximum amplitude before and after the cold pressor test was evaluated at $22.9 \%$ for the systolic wave and at $33.2 \%$ for the dicrotic notch Then, a very high frame rate imaging (15000 FPS) was performed on one healthy subject. The results obtain on the 2D wall motion estimation allowed us to measure for the first time the longitudinal motion propagation of an in vivo carotid wall. Its mean velocity was evaluated at $16.1 \mathrm{~m} . \mathrm{s}^{-1}$, which is approximately 3 times higher than the estimated PWV.

\section{ACKNOWLEDGMENT}

This work was performed within the framework of the LABEX CELYA (ANR-10-LABX-0060) and Labex PRIMES (ANR-10-LABX-0063) of Université de Lyon, within the program "Investissements d'Avenir" (ANR-11-IDEX-0007) operated by the French Nation-al Research Agency (ANR).

\section{REFERENCES}

[1] M. Tanter and M. Fink, "Ultrafast imaging in biomedical ultrasound," IEEE Trans. Ultrason. Ferroelectr. Freq. Control, vol. 61, no. 1, pp. 102-119, Jan. 2014

[2] J. Provost, V. Gurev, N. Trayanova, and E. E. Konofagou, "Mapping of cardiac electrical activation with electromechanical wave imaging: an in silico-in vivo reciprocity study," Heart Rhythm Off. J. Heart Rhythm Soc., vol. 8, no. 5, pp. 752-759, May 2011.

[3] J. Udesen, F. Gran, K. L. Hansen, J. A. Jensen, C. Thomsen, and M. B. Nielsen, "High frame-rate blood vector velocity imaging using plane waves: simulations and preliminary experiments," IEEE Trans. Ultrason. Ferroelectr. Freq. Control, vol. 55, no. 8, pp. 1729-1743, Aug. 2008.

[4] M. Cinthio, A. R. Ahlgren, T. Jansson, A. Eriksson, H. W. Persson, and K. Lindström, "Evaluation of an ultrasonic echo-tracking method for measurements of arterial wall movements in two dimensions," IEEE Trans. Ultrason. Ferroelectr. Freq. Control, vol. 52, no. 8, pp. 13001311, Aug. 2005.

[5] G. Zahnd, L. Boussel, A. Marion, M. Durand, P. Moulin, A. Sérusclat, and D. Vray, "Measurement of two-dimensional movement parameters of the carotid artery wall for early detection of arteriosclerosis: a preliminary clinical study," Ultrasound Med. Biol., vol. 37, no. 9, pp. 1421-1429, Sep. 2011.
[6] M. Couade, M. Pernot, C. Prada, E. Messas, J. Emmerich, P. Bruneval, A. Criton, M. Fink, and M. Tanter, "Quantitative assessment of arterial wall biomechanical properties using shear wave imaging," Ultrasound Med. Biol., vol. 36, no. 10, pp. 1662-1676, Oct. 2010.

[7] M. Shinohara, K. Sabra, J.-L. Gennisson, M. Fink, and M. Tanter, "Real-time visualization of muscle stiffness distribution with ultrasound shear wave imaging during muscle contraction," Muscle Nerve, vol. 42, no. 3, pp. 438-441, Sep. 2010.

[8] J. Provost, A. Gambhir, J. Vest, H. Garan, and E. E. Konofagou, "A clinical feasibility study of atrial and ventricular electromechanical wave imaging," Heart Rhythm Off. J. Heart Rhythm Soc., vol. 10, no. 6, pp. 856-862, Jun. 2013.

[9] K. Fujikura, J. Luo, V. Gamarnik, M. Pernot, R. Fukumoto, M. Tilson, and E. E. Konofagou, "A novel noninvasive technique for pulse-wave imaging and characterization of clinically-significant vascular mechanical properties in vivo," Ultrason. Imaging, vol. 29, no. 3, pp. 137-154, Jul. 2007.

[10] R. L. Van Citters, "Longitudinal waves in the walls of fluid-filled elastic tubes," Circ. Res., vol. 8, pp. 1145-1148, Nov. 1960.

[11] P. Flaud, D. Geiger, C. Oddou, and D. Quémada, "Écoulements pulsés dans les tuyaux viscoélastiques. Application a l'étude de la circulation sanguine," J. Phys., vol. 35, no. 11, pp. 869-882, 1974.

[12] M. Anliker, W. E. Moritz, and E. Ogden, "Transmission characteristics of axial waves in blood vessels," J. Biomech., vol. 1, no. 4, pp. 235-246, Dec. 1968.

[13] J. A. Jensen and P. Munk, "A new method for estimation of velocity vectors," IEEE Trans. Ultrason. Ferroelectr. Freq. Control, vol. 45, no. 3, pp. 837-851, 1998.

[14] M. E. Aderson, "Multi-dimensional velocity estimation with ultrasound using spatial quadrature," IEEE Trans. Ultrason. Ferroelectr. Freq. Control, vol. 45, no. 3, pp. 852-861, May 1998.

[15] H. Liebgott, J. Fromageau, J. E. Wilhjelm, D. Vray, and P. Delachartre, "Beamforming Scheme for 2D Displacement Estimation in Ultrasound Imaging," EURASIP J. Adv. Signal Process., vol. 2005, no. 8, p. 932740, 2005.

[16] H. Liebgott, A. Basarab, P. Gueth, D. Friboulet, and P. Delachartre, "Transverse oscillations for tissue motion estimation," Ultrasonics, vol. 50, no. 6, pp. 548-555, May 2010.

[17] T. Shiina, K. Kondo, and M. Yamakawa, "Displacement vector measurement based on two-dimensional modulation method with hyperbolic scanning," in Ultrasonics Symposium (IUS), 2009 IEEE International, 2009, pp. 2284-2287.

[18] C. Sumi, "Displacement vector measurement using instantaneous ultrasound signal phase - multidimensional autocorrelation and doppler methods," IEEE Trans. Ultrason. Ferroelectr. Freq. Control, vol. 55, no. 1, pp. 24-43, Jan. 2008.

[19] A. Basarab, P. Gueth, H. Liebgott, and P. Delachartre, "Phase-based block matching applied to motion estimation with unconventional beamforming strategies," IEEE Trans. Ultrason. Ferroelectr. Freq. Control, vol. 56, no. 5, pp. 945-957, May 2009.

[20] G. Zahnd, S. Salles, H. Liebgott, D. Vray, A. Sérusclat, and P. Moulin, "Real-time ultrasound-tagging to track the 2D motion of the common carotid artery wall in vivo," Med. Phys., vol. 42, no. 2, pp. 820-830, Feb. 2015.

[21] S. Salles, H. Liebgott, D. Garcia, and D. Vray, "Full 3-D transverse oscillations: a method for tissue motion estimation," IEEE Trans. Ultrason. Ferroelectr. Freq. Control, vol. 62, no. 8, pp. 1473-1485, Aug. 2015.

[22] S. Salles, A. J. Y. Chee, D. Garcia, A. C. H. Yu, D. Vray, and H. Liebgott, "2-D arterial wall motion imaging using ultrafast ultrasound and transverse oscillations," IEEE Trans. Ultrason. Ferroelectr. Freq. Control, vol. 62, no. 6, pp. 1047-1058, Jun. 2015.

[23] D. Garcia, L. Le Tarnec, S. Muth, E. Montagnon, J. Porée, and G. Cloutier, "Stolt's f-k migration for plane wave ultrasound imaging," IEEE Trans. Ultrason. Ferroelectr. Freq. Control, vol. 60, no. 9, pp. 1853-1867, Sep. 2013.

[24] K. Moriyama and H. Ifuku, "Increased cardiovascular reactivity to the cold pressor test is not associated with increased reactivity to isometric handgrip exercise," Eur. J. Appl. Physiol., vol. 108, no. 4, pp. 837-843, Mar. 2010.

[25] V. R. Bellinazzi, A. C. Sposito, R. Schreiber, J. G. Mill, J. E. Krieger, A. C. Pereira, and W. Nadruz, "Response to Cold Pressor Test Predicts Long-Term Changes in Pulse Wave Velocity in Men," Am. J. Hypertens., vol. 27, no. 2, pp. 157-161, Feb. 2014. 